\title{
Energy Aware Transmission in Cellular Uplink with Clustered Base Station Cooperation
}

\author{
Efstathios Katranaras and Muhammad Ali Imran \\ Centre for Communication Systems Research, CCSR \\ University of Surrey, UK \\ Email: e.katranaras@surrey.ac.uk
}

\author{
Reza Hoshyar \\ National Semiconductor \\ California, USA \\ Email: reza_hoshyar@yahoo.com
}

\begin{abstract}
We provide an analytical formula to evaluate the performance of the uplink of planar cellular networks when joint processing is enabled among limited number of base stations in a generalised fading environment. Focusing on user transmission power allocation techniques to mitigate inter-cluster interference we investigate the system's spectral-energy efficiency trade-off. The paper addresses the gains in both cell throughput and transmissions energy efficiency due to the combined strategies of base station cooperation and user power management. We assess the effect of the propagation environment and of the key network design parameters of cooperation cluster size and intersite distance on the overall performance providing numerical results for a real-world scenario.
\end{abstract}

\section{INTRODUCTION}

Communication systems' increasingly demand for high quality and throughput services leads industry into aggressive radio spectrum usage. Significant losses in throughput and fairness degradation though occur due to increased amount of intercell interference (ICI) in current cellular systems. That has rendered Coordinated Multi Point (CoMP) a promising technology to mitigate and even exploit ICI through signal joint processing, reception and/or transmission at the Base Stations (BSs) and has the potential to boost spectral efficiency as well as to provide more homogeneous user data rates distribution [1], [2].

On the other hand, energy consumption of communication technologies is of great interest recently for both environmental and economical reasons [3], [4]. The energy efficiency assessment of auspicious technologies like CoMP will serve as a useful tool to investigate the trade-off between the gains in throughput and the energy consumption in cellular networks. CoMP requires additional infrastructural cost, low latency backhaul links between BSs, signalling overheads and signal processing at the transceivers end. However, BS cooperation provides the potential to save energy due to less signal power needed from the Signal to Interference plus Noise Ratio (SINR) gains offered. This work targets to identify efficient User Terminal (UT) trasnmit power allocations to conserve energy and improve performance in CoMP enabled systems.

In practical systems only a limited number of BSs can cooperate in order for the inter-base communication overhead to be affordable. Numerous works recently addressed that clustered cooperation scheme [5], [6]. Moreover, in previous work on a linear cellular model, we also adopted the clustered joint processing strategy investigating various cluster isolation techniques [7], [8]. However, the focus of the aforementioned research is restricted mostly in the evaluation of the throughput performance. In this paper we move one step forward and considering the uplink of a planar cellular system we address the effects of the CoMP technology on total energy consumption. Then we investigate in depth the trade-off between the achieved spectral efficiency and the potential energy gains due to energy aware transmission.

The rest of the paper is organised as follows. In Section II the system model is introduced. In Section III we first focus on the ergodic achievable sum rate performance and then turn onto the energy efficiency analysis of the problem. In Section IV we analyse the transmit power management approach and integrate it with the performance analysis. Section V provides a numerical representation for a real-world scenario of the results obtained by the analysis along with insightful observations. Finally, Section VI concludes the paper.

Notation: $\mathbb{E}[\cdot]$ stands for the expectation of the respective variable or matrix and $\boldsymbol{\Lambda}_{(\cdot)}$ stands for the covariance matrix of the respective vector. $\mathcal{C N}$ represents a complex Gaussian distribution. $\mathbb{Z}_{n}$ is the finite set of integers modulo $n$. Symbols $\triangleq$ and $\underset{n \rightarrow \infty}{\stackrel{a . s .}{\longrightarrow}}$ stand for "is defined as" and "converges almost surely when $n$ tends to infinity", respectively.

\section{SySTEM MODEL}

A planar cellular array is considered, similar to the one presented in [9], focusing on the uplink. BSs, each one at the center of a hexagonal cell, are uniformly distributed over the 2D grid. $K$ UTs are uniformly distributed over each cell. The network of a total of $N$ cells is divided into $M$ sets of cells, grouped together to form clusters of size $Q$, i.e. $N=M Q$, with $Q \ll N$. Due to the restrictions of hexagonal geometry, not all cluster sizes are possible. Here, without loss of generality, we direct our analysis on the most geometrically tractable cases, i.e. $Q=3,4$ and $\left(1+\sum_{l}^{L_{Q}} 6 l\right)$, where $l=0,1, . ., L_{Q}$ and $L_{Q}$ stands for the total number of rings of cells to form each cluster.

The cooperation among BSs is limited only to those in cells that belong to the same cluster and hence a Joint Processor (JP) in each cluster combines all the UTs' received signals of that cluster. An inter-cluster interference allowance scheme [7] with 
no spectral isolation between clusters is considered and thus, all UTs are allowed to exploit the full resources allocated to the system. Every cluster experiences inter-cluster interference due to transmitted signals from UTs in other clusters.

\section{A. Propagation Model}

The signal propagation model presented in [9] is also employed here. Path attenuation is related to distance through a power-law model as:

$$
\varsigma_{k, \dot{q}, \dot{m}}^{m, q}=\sqrt{L_{0}}\left(1+d_{\dot{m}, \dot{q}, k}^{m, q}\right)^{-\eta / 2}
$$

where $L_{0}$ specifies the power received at a unit reference distance for unit transmit power and $\eta$ denotes the path loss exponent. $d_{\dot{m}, \dot{q}, k}^{m, q}$ is defined as the distance between user $k$ in cell $\dot{q}$ of cluster $\dot{m}$ from the reference circle, essentially co-located with the BS, in cell $q$ of cluster $m$. Moreover, a generalised model for multipath fading is given by [10]:

$$
g_{\dot{m}, \dot{q}, k}^{m, q}=\sqrt{\frac{\kappa}{\kappa+1}} \mathrm{e}^{j \Phi_{\dot{m}, \dot{q}, k}^{m, q}}+\sqrt{\frac{1}{\kappa+1}} \mathcal{C N}(0,1)
$$

where $\Phi_{\dot{m}, \dot{q}, k}^{m, q}$ is the independent, uniformly distributed random received phase on the specular path between transmitter $k$ in cell $\dot{q}$ of cluster $\dot{m}$ and the BS of cell $q$ of cluster $m, \kappa$ is the power ratio of the specular path and the non-specular multipaths and $\mathbb{E}[g g *]=1$. Finally, it is noted that the effects of shadow fading can be captured by parameters $L_{0}$ and $\eta$ [9].

\section{B. Channel Model}

Assume symmetry among all clusters of cells, e.g. a toroidal array model can be considered to avoid the system edge effects. In that case, results from single cluster analysis will be valid for the whole system. Hence, the received signal $y^{m, q}$ at the BS in cell $q \in \mathbb{Z}_{Q}$ of any cluster $m \in \mathbb{Z}_{M}$ is the sum of the transmitted signals $x_{m, \dot{q}, k}$ of all the UTs in the same cluster of cells appropriately scaled by each channel attenuation coefficient, plus the sum $z^{m, q}$ of the thermal AWGN $\left(n^{m, q}\right.$ with $\left.n \sim \mathcal{C N}\left(0, \sigma^{2}\right)\right)$ and the interfering signals from UTs in cells outside the cluster of interest:

$$
y^{m, q}=\sum_{\dot{q}=1}^{Q} \sum_{k=1}^{K} \varsigma_{m, \dot{q}, k}^{m, q} g_{m, \dot{q}, k}^{m, q} x_{m, \dot{q}, k}+z^{m, q}
$$

Inter-cluster interference, can be assumed a sum of complex Gaussian inputs and thus, can be viewed as an additional AWGN component at the BSs. Hence, the power of the undesired signal $z^{m, q}=n^{m, q}+$ $\sum_{\dot{m} \neq m} \sum_{\dot{q}=1}^{Q} \sum_{k=1}^{K}\left[\varsigma_{\dot{m}, \dot{q}, k}^{m, q} g_{\dot{m}, \dot{q}, k}^{m, q} x_{\dot{m}, \dot{q}, k}\right]$ can be given by

$$
\begin{aligned}
\mathbb{E}\left[z^{m, q}\left(z^{m, q}\right)^{*}\right]=\sigma^{2}+\sum_{\dot{m}} \sum_{\dot{q}=1}^{Q} \sum_{k=1}^{K} \\
\mathbb{E}\left[\left(\varsigma_{\dot{m}, \dot{q}, k}^{m, q} g_{\dot{m}, \dot{q}, k}^{m, q} x_{\dot{m}, \dot{q}, k}\right)\left(\varsigma_{\dot{m}, \dot{q}, k}^{m, q} g_{\dot{m}, \dot{q}, k}^{m, q} x_{\dot{m}, \dot{q}, k}\right)^{*}\right]
\end{aligned}
$$

\section{Spectral AND ENERGy EfFiciency}

In wireless communications traditionally spectrum and recently power have been considered as the scarcest resources consumed to attain the most basic utility of information exchange. Thus, the spectral-energy efficiency trade-off becomes an important performance measure in the design of a cellular system. Future solutions should target to carefully utilize the available energy subject to a required number of bits to be delivered over a certain time and in a given bandwidth. In this paper we consider the per cluster ergodic achievable sum rate to evaluate the spectral efficiency of the system. On the other hand, energy efficiency is characterized by focusing on the efficient management of the UTs transmit power consumption.

\section{A. Ergodic Achievable Cluster Sum Rate}

Consider $\mathbf{x}=\left[\mathbf{x}_{m, 1}{ }^{T}, \mathbf{x}_{m, 2}{ }^{T}, \ldots \mathbf{x}_{m, Q^{T}}\right]^{T}$ to be the $Q K \times 1$ vector of the transmitted signals of all the UTs in cluster $m$, with $\mathbf{x}_{m, q}=\left[x_{m, q, 1}, \ldots, x_{m, q, K}\right]^{T}$ denoting the concatenation of the transmitted signals from the $K$ UTs in cell $q, \mathbf{z}$ the $Q \times 1$ noise vector and $\mathbf{H}$ the overall $Q \times Q K$ cluster channel gain matrix. For large number of users per cell, a tight upper bound for the ergodic achievable cluster sum rate is obtained from the strong law of large numbers [7], [2]:

$$
\begin{aligned}
& \mathrm{SE}_{Q} \triangleq R=\mathbb{E}_{\mathbf{H}}\left[\log \left(\frac{\operatorname{det}\left(\mathbf{H} \boldsymbol{\Lambda}_{\mathbf{x}} \mathbf{H}^{\dagger}+\boldsymbol{\Lambda}_{\mathbf{z}}\right)}{\operatorname{det}\left(\boldsymbol{\Lambda}_{\mathbf{z}}\right)}\right)\right] \\
& \underset{K \rightarrow \infty}{\stackrel{a . s .}{\longrightarrow}} \log \left(\frac{\operatorname{det} \mathbb{E}_{\mathbf{H}}\left[\mathbf{H} \boldsymbol{\Lambda}_{\mathbf{x}} \mathbf{H}^{\dagger}+\boldsymbol{\Lambda}_{\mathbf{z}}\right]}{\operatorname{det} \mathbb{E}_{\mathbf{H}}\left(\boldsymbol{\Lambda}_{\mathbf{z}}\right)}\right) \text { bits/sec/Hz }
\end{aligned}
$$

where the expectation is taken over all the system fading realizations.

\section{B. UT Transmit Energy Efficiency}

The CoMP strategy in uplink eventually results in higher SINR which translates to increased UTs spectral efficiency and potential savings on the overall network energy resource when an operator is interested in satisfying certain rate constraints. However, additional energy is required to maintain a successful BS cooperation scheme. A general model for the power consumption in CoMP enabled systems can be given by:

$$
P_{\mathrm{CoMP}}=P_{\mathrm{SCP}}+\Delta P_{\mathrm{Bh}}+\Delta P_{\mathrm{PP}}-\Delta P_{\mathrm{Tx}}
$$

where $P_{\mathrm{SCP}}, \Delta P_{\mathrm{Bh}}, \Delta P_{\mathrm{PP}}$ and $\Delta P_{\mathrm{Tx}}$ stand for the consumed power of the conventional signal cell processing scheme, the power needs for the extra backhauling, the additional BS processing power needs and the savings on radiated power from transmissions, respectively. The study of all power elements in (6) on both uplink and downlink channel is essential for a complete evaluation on the energy efficiency of the CoMP scheme. In that regard, some initial work on BS energy consumption is presented in [11]. In this paper though, our interest lies on identifying and evaluating the efficiency of UTs power management strategies under CoMP. Thus, we put aside the consideration of energy dissipation at BSs and we focus on the $\Delta P_{\mathrm{Tx}}$ conservations. 
Hence, the system average transmit energy efficiency can be given, similarly to Verdu's energy per bit definition in [12] for the single channel case, as:

$$
\mathrm{EE}_{Q} \triangleq \frac{R}{\sum_{q} \sum_{k} P_{q, k}} \text { bits/Hz/Joule }
$$

where $P_{q, k}$ denotes the transmitted signal power of UT $k$ in cell $q$ of any cluster.

\section{UT Power Management For Cluster Isolation}

An efficient way to reduce both inter-cluster interference and system transmit energy consumption is to perform power control on the UTs' transmissions according to the interference each one causes on neighbouring clusters. In [13] it was shown that in CoMP deployments cell-edge UTs (or more generally, UTs in a disadvantageous channel situation) under any decoding technique contribute the least on the total sum rate. Based on these findings, in [8] for the linear system case, it was heuristically shown that a UT power allocation profile as a function of the instantaneous position of each UT in respect to its allocated BS was achieving high sum rates especially for systems that are not very dense (where spectral cluster isolation techniques may be preferable [7]) neither very sparse (where CoMP techniques have no actual effect). The optimization analysis also revealed that the optimal UT power profile has to be a decreasing function of the UT distance from the center of its respective cell and cluster.

\section{A. Cell-based Power Allocation}

Here, we also adopt that Cell-based UT Power Allocation profile (CPA) which can provide a simple and tractable, yet insightful analysis of the power management problem. In CPA scheme (Figure 1a, 1c) the transmitted power of a UT will be a linear function of distance $s$ from its associated BS:

$$
P_{q, k}=\left\{\begin{array}{cc}
P_{\min } & \left(1-\alpha_{2}\right) D_{0} \leq s \leq D_{0} \\
P(s) & \alpha_{1} D_{0} \leq s \leq\left(1-\alpha_{2}\right) D_{0} \\
P_{\max } & 0 \leq s \leq \alpha_{1} D_{0}
\end{array}\right\}, \forall q
$$

where $D_{l}=\frac{6}{\pi} \int_{0}^{\frac{\pi}{6}} \frac{r_{l}}{\cos \theta} d \theta$, with $l \in \mathbb{N}$, stands for the equivalent, to the hexagonal cell with side $r_{l}$, circular cell radius and $P(s) \triangleq P_{\min }+\left(P_{\max }-P_{\min }\right) \frac{\left(1-\alpha_{2}\right) D_{0}-s}{\left(1-\alpha_{1}-\alpha_{2}\right) D_{0}}$. Note that the minimum power constraint ensures that all UTs are able to perform their basic and emergency communication needs at any time.

Assuming independent transmitted signals from the UTs in each cell, we have:

$$
\boldsymbol{\Lambda}_{\mathbf{x}}=\operatorname{diag}(\overbrace{P_{1,1}, \ldots, P_{q, k}, \ldots, P_{Q, K}}^{Q K \text { elements }})
$$

and

$$
\mathbf{\Lambda}_{\mathbf{z}}=\operatorname{diag}(\overbrace{Z_{1}, \ldots, Z_{q}, \ldots, Z_{Q}}^{Q \text { elements }})
$$

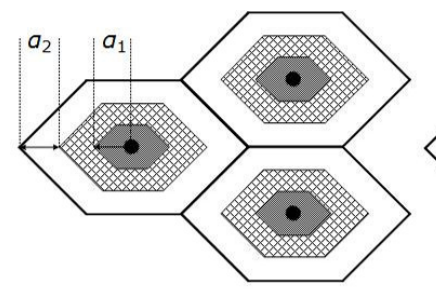

(a)

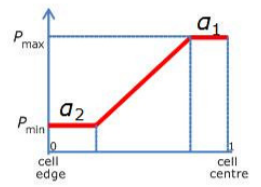

Fig. 1. UT Power management example $(Q=3)$. UTs in edge areas transmit with $P_{\min }$, in centre areas with $P_{\max }$ and rest UTs follow a linear power profile. (a) Cell-based power allocation, (b) Cluster-edge-based power allocation, (c) UT power allocation as function of UT position within cell.

where $Z_{q} \triangleq \sigma^{2}+\sum_{\dot{m}} \sum_{\dot{q}=1}^{Q} \sum_{k=1}^{K} P_{\dot{q}, k}\left(\varsigma_{\dot{m}, \dot{q}, k}^{m, q}\right)^{2}$. The expected product of two different realisations of the fading coefficients $\mathbb{E}_{g}\left[g(\dot{g})^{*}\right]=0$ and thus, $\mathbb{E}_{\mathbf{H}}\left[\mathbf{H} \boldsymbol{\Lambda}_{\mathbf{x}} \mathbf{H}^{\dagger}\right]$ converges to a $Q \times Q$ diagonal matrix simplifying (5) to:

$$
R \underset{K \rightarrow \infty}{\stackrel{a . s .}{\longrightarrow}} \log \prod_{q=1}^{Q}\left[1+\frac{\sum_{\dot{q}=1}^{Q} \sum_{k=1}^{K} P_{k}\left(\varsigma_{m, \dot{q}, k}^{m, q}\right)^{2}}{\sigma^{2}+\sum_{\dot{m}} \sum_{\dot{q}=1}^{Q} \sum_{k=1}^{K} P_{k}\left(\varsigma_{\dot{m}, \dot{q}, k}^{m, q}\right)^{2}}\right]
$$

\section{B. Cluster-based Power Allocation}

Since inter-cluster interference is mostly originated from UTs located closer to cluster edges, a meaningful power allocation would be to manage the power of UTs that are only at cluster edges according to the power profile in (8) while all other UTs are allowed to transmit at $P_{\max }$. This cluster-Edgebased Power Allocation scheme (EPA) is illustrated in Figure $1 \mathrm{~b}$ for the case of $Q=3$. An immediate intuition for EPA is that it shall achieve higher sum rates than CPA since UTs at the centre of the cluster, which experience less inter-cluster interference, can achieve high SINR. On the other hand, as the cluster size increases, a lower percentage of UTs in the cluster manage their transmitting power and the energy savings are reduced. Thus, it will be very interesting to evaluate which effect becomes stronger in that case. During the presentation of the numerical results we will compare and provide insight on the spectral-energy efficiency trade-off for both cell- and cluster-edge-based power allocation paradigms.

\section{NUMERICAL RESUltS AND OBSERVATIONS}

To numerically evaluate the system performance, we need a more tractable analytical formula than the one provided in (11). For that reason, we adopt the average path loss approximation approach presented in [9] and we produce numerical results based on the approximated cluster sum rate 


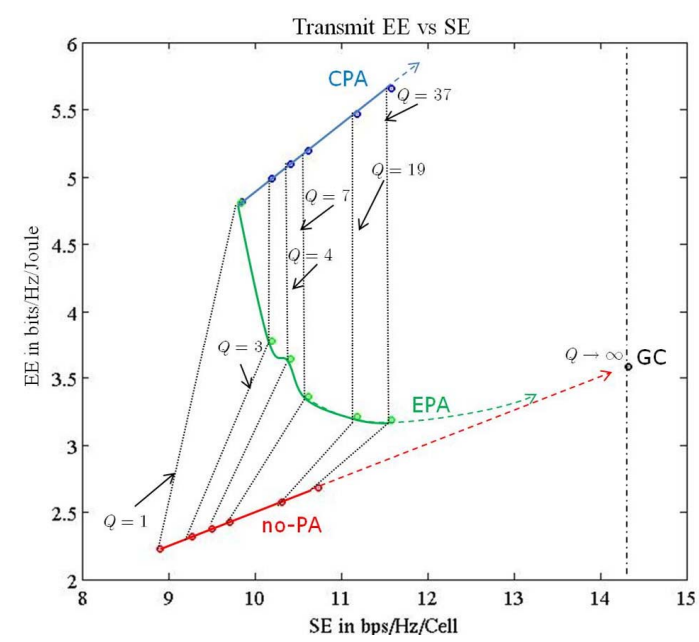

Fig. 2. SE versus transmit EE for various power management strategies and cluster sizes. ISD $=2 \mathrm{Km}, \eta=3, \alpha_{1}=0.1, \alpha_{2}=0.8$.

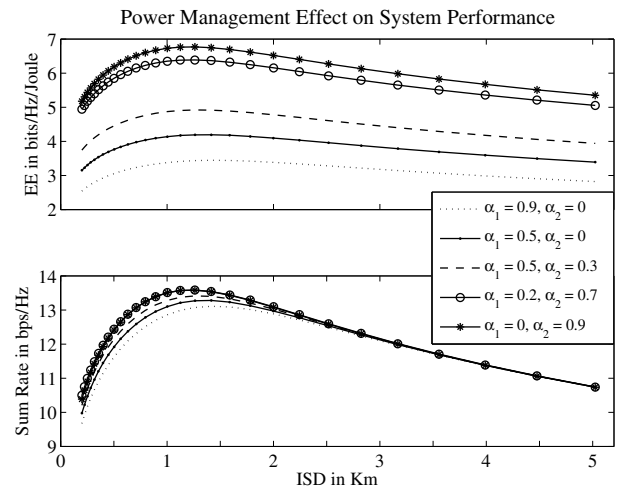

Fig. 3. Transmit EE and average cell Sum Rate versus ISD for different values of $\alpha_{1}$ and $\alpha_{2} . Q=7, \eta=3.5$.

which converges to $R$ for large enough $K$ :

$$
R_{\text {approx. }} \triangleq \log \prod_{q=1}^{Q}\left[1+\frac{K \sum_{\dot{q}=1}^{Q} \overline{\zeta_{m, \dot{q}}^{m, q}}}{\sigma^{2}+K \sum_{\dot{m}} \sum_{\dot{q}=1}^{Q} \overline{\zeta_{\dot{m}, \dot{q}}^{m, q}}}\right]
$$

where $\overline{\zeta_{\dot{m}, \dot{q}}^{m, q}}=\frac{P_{\dot{q}}}{\pi D^{2}} \int_{0}^{D} \int_{-\pi}^{\pi} \frac{L_{0}}{\left(1+d_{l, k}(\theta, s)\right)^{\eta}} s d \theta d s$ with $d_{l, k}(\theta, s)=\sqrt{\left(\frac{D_{l}+D_{l-1}}{2}-s \sin \theta\right)^{2}+(s \cos \theta)^{2}} \cdot P_{\dot{q}}$ denotes the overall transmitted power from all UTs in cell $\dot{q}$ and can be obtained from the power profile given in (8) as the volume of a truncated cone, i.e. $P_{\dot{q}}=$ $P_{\min }+\frac{1}{3}\left(P_{\max }-P_{\min }\right)\left(\alpha_{1}^{2}+\left(1-\alpha_{2}\right)^{2}+\alpha_{1}\left(1-\alpha_{2}\right)\right)$. Note also that subscript $l$ defines the number of tiers that cell $\dot{q}$ in cluster $\dot{m}$ is far from cell $q$ in cluster $m$.

For interpreting the information theoretic results into realworld systems we assume the practical scenario described in [9] with the empirical value of $L_{0}$ to be $-34.5 \mathrm{dBW}$. UT power constraints are $P_{\min }=100 \mathrm{~mW}$ and $P_{\max }=200 \mathrm{~mW}$. The results in Figures 2-4 have been produced for planar cellular systems which contain equivalent clusters of $1,3,4,7,19$ and 37 cells respectively, considering a toroidal-like model with always 10 tiers of cells around each cell to avoid edge effects.
20 uniformly distributed UTs over each cell are considered. For presenting the results characterizing the system, we calculate the average, over the cluster size, cell sum rate (SE in bps/Hz/Cell) and the respective, as given in (7), transmit energy efficiency (EE in bits/Hz/Joule).

In Figure 2, we plot the performances of the CPA and EPA schemes in comparison to the one achieved without any power allocation strategy (no-PA scheme). A medium density system with Inter-Site Distance (ISD) of $2 \mathrm{Km}$ and $\eta=3$ is considered. Power allocation parameters $\alpha_{1}=0.1$ and $\alpha_{2}=0.8$ were chosen as it was observed to provide substantial performance gain. The benchmark performance of the system under global cooperation (GC), where all BSs cooperate to combine received signals providing the upper limit for the achievable sum rate of the given cellular system, is also illustrated. We observe that both CPA and EPA achieve similar sum rates and outperform the no-PA scheme since the power management and the resulted mitigation of the intercluster interference translate into both increased spectral and energy efficiency. It also becomes apparent that CPA is the most energy efficient scheme. That is explained from the fact that in CPA we restrain the power of cell edge UTs whose rates are anyway exceptionally low when summing up to the cell sum rate. Finally, it is worth noting that higher cooperation, in the form of higher cluster size $Q$, provides gain in both SE and EE for CPA and no-PA schemes. On the other hand, with increasing $Q$, the EE of EPA boils eventually down to the EE of no-PA since the power conservations which are performed only at a portion of the cluster edge cells become minimal.

Figures 3 and 4 focus on the high EE achieving CPA scheme. It is worth noting here that in CPA (as well as in no-PA scheme) the relationship between SE and EE is linear since the average cell power remains constant independently of the size of the cluster. Figure 3 illustrates the effect of UT power management on system performance. Transmit energy efficiency and average cell sum rate are respectively plotted against ISD for various power allocation schemes, i.e. different $\left\{\alpha_{1}, \alpha_{2}\right\}$ combinations. A number of interesting observations is depicted here. First, we notice that power management offers a gain in SE only for relatively small ISDs. This is expected since inter-cluster interference becomes less significant for systems with large ISD and thus there is less need for power allocation in that case. The gain in SE is minor when compared to the one achieved in EE. Unlike the SE's case, EE is boosted by power management almost equally for any system ISD. Moreover, we observe that the gain increases 1) when less UTs transmit with $P_{\max }, 2$ ) when more edge users transmit with $P_{\min }$ and c) when the system has the appropriate density. Residing on the last observation, we note that there exists a specific ISD that maximizes the EE (and equivalently the cluster sum rate) of the system. Smaller ISD results to larger inter-cluster interference and lower performance (interferencelimited regime). Additionally, systems with larger ISD do not benefit at maximum from the BS cooperation scheme and also underperform (noise-limited regime).

In Figure 4, the combined effect of cell density and path 


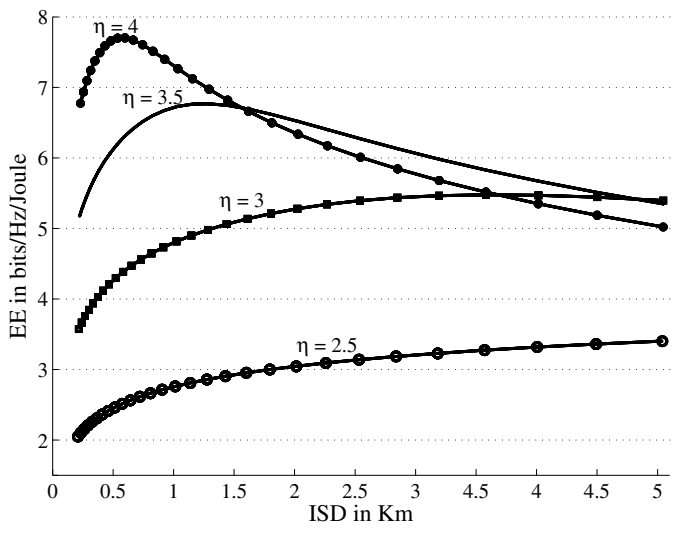

Fig. 4. Transmit EE versus ISD for various path loss exponent values. $Q=7$, $\alpha_{1}=0, \alpha_{2}=0.9$.

loss exponent (termed throughout this paper as system density) on the EE is investigated. EE is plotted for various density systems $(\mathrm{ISD}=200 \mathrm{~m}-5 \mathrm{Km}, \eta=2.5,3,3.5,4)$ cooperating with $Q=7$ while $\alpha_{1}=0$ and $\alpha_{2}=0.9$. It is observed that the ISD value that maximizes the EE depends on the path loss exponent. Larger $\eta$ moves the optimal performance to the left of the ISD axis, which translates to the fact that relatively denser systems (but not too dense where inter-cluster interference becomes dominant overcoming the power management technique) may become more energy efficient due to the BS cooperation and UT power management combined strategies.

\section{A. Discussion}

In the previous section we perceived that more energy can be conserved if UTs transmit with the minimum power allowed when under dire channel conditions, i.e. when at any time being located close enough to the edge of their cell/cluster or more generally when in deep fading situation. It shall be noted that our analysis may be applied to a practical system where the UT power allocation is updated constantly based on the instantaneous UT signal strength. Our consideration here that signal strength is a straightforward function of distance can be further extended by including the channels slow and the instantaneous fast fading effect for the UTs power usage decision making without any loss of generality.

However, the proposed combined strategy of clustered cooperation with UT power management requires a non-greedy transmission approach from the UTs end. In other words, this research is more appropriate to be considered for delay tolerant applications and/or in mobile scenarios. In those cases, UTs which are in a disadvantageous signal situation have the capability to delay their data transmissions and seize the opportunity when their SNR becomes strong enough. In that way, over a large number of fading and/or topology realisations, our analysis results become more valid for both the long-term average cluster sum rate and the UT transmit energy efficiency.

\section{CONCLUSION}

In this paper we assessed the spectral and energy efficiency of CoMP enabled systems. We focused on the gains achieved from implementing a power management strategy on the UTs' transmission powers and we observed how key system parameters affect the overall performance. The main findings of the paper can be concluded as follows. 1) Under a clustered BS cooperation scheme with inter-cluster interference there is an optimal ISD value, depending on the propagation environment, for which performance is maximized. 2) UT power management for inter-cluster interference mitigation can provide a marginal gain on the spectral efficiency of high density systems. More significant though is the gain provided in energy efficiency for any kind of density system. 3) The CPA scheme with most non-greedy UTs transmitting at $P_{\min }$ and only few central UTs allowed to transmit at $P_{\max }$ proves to be the most performance efficient strategy even for small number of cooperating BSs. This work can be a starting point for a further research to include the energy consumption at the radio access network side on both uplink and downlink and result into a generalised framework for spectral-energy efficiency trade-off evaluation in CoMP enabled cellular systems.

\section{REFERENCES}

[1] M. Karakayali, G. Foschini, and R. Valenzuela, "Network coordination for spectrally efficient communications in cellular systems," Wireless Communications, IEEE [see also IEEE Personal Communications], vol. 13, no. 4, pp. 56-61, Aug. 2006.

[2] O. Somekh, O. Simeone, Y. Bar-Ness, A. Haimovich, U. Spagnolini, and S. S. (Shitz), Distributed Antenna Systems: Open Architecture for Future Wireless Communications.

[3] G. Auer, I. Godor, L. Hevizi, M. A. Imran, J. Malmodin, P. Fazekas, G. Boczok, D. Zeller, O. Blume, and R. Tafazolli, "The EARTH Project: Towards Energy Efficient Wireless Networks," ICT Future Network and Mobile Summit, June 2010.

[4] G. P. Fettweis and E. Zimmermann, "ICT energy consumption - trends and challenges," in Proceedings of the 11th International Symposium on Wireless Personal Multimedia Communications, July 2008.

[5] A. Papadogiannis, D. Gesbert, and E. Hardouin, "A Dynamic Clustering Approach in Wireless Networks with Multi-Cell Cooperative Processing," IEEE International Conference on Communications, (ICC'08), 2008.

[6] S. Venkatesan, "Coordinating Base Stations for Greater Uplink Spectral Efficiency in a Cellular Network," IEEE 18th International Symposium on Personal, Indoor and Mobile Radio Communications (PIMRC'07), 2007.

[7] E. Katranaras, M. Imran, and R. Hoshyar, "Sum Rate of Linear Cellular Systems with Clustered Joint Processing," IEEE 69th Vehicular Technology Conference, (VTC'09), 2009.

[8] E. Katranaras, D. Kaltakis, M. Imran, and R. Hoshyar, "Interference Allowance in Clustered Joint Processing and Power Allocation," 6th International Wireless Communications and Mobile Computing Conference, (IWCMC'10), 2010.

[9] E. Katranaras, M. Imran, and C. Tzaras, "Uplink Capacity of a Variable Density Cellular System with Multicell Processing," IEEE Transactions on Communications, vol. 57, no. 7, pp. 2098-2108, July 2009.

[10] D. Tse and P. Viswanath, Fundamentals of Wireless Communications. University Press, Cambridge, 2005.

[11] O. Arnold, F. Richter, G. Fettweis, and O. Blume, "Power consumption modeling for different base station types in heterogeneous cellular networks," Future Network and Mobile Summit, 2010.

[12] S. Verdu, "Spectral efficiency in the wideband regime," Information Theory, IEEE Transactions on, vol. 48, no. 6, pp. 1319-1343, 2002.

[13] D. Kaltakis, M. Imran, E. Katranaras, and R. Hoshyar, "Fairness and User Rate Distribution in Joint Processing Systems," IEEE International Conference on Communications, (ICC'10), 2010. 